\title{
Duet Lectorials: An Engaging Approach to Interdisciplinary Teaching
}

\author{
Slattery, Robin Marie ${ }^{a}$; Taylor, Renea .Anne. ${ }^{b}$ and Doerig, Christian Daniel ${ }^{\mathrm{c}}$
}

${ }^{a}$ Department of Immunology, Central Clinical School, Monash University, Australia.

${ }^{b}$ Department of Physiology, Monash University, Australia. ${ }^{c}$ Department of Microbiology, School of Biomedical Science, Faculty of Medicine, Nursing and Health Sciences, Monash University, Australia.

\begin{abstract}
Duet Lectorials were delivered to third-year students undertaking their final 'cap-stone' unit by two experts straddling two inter-related but distinct biomedical disciplines. This interdisciplinary teaching approach was introduced for two reasons: firstly, to address a gap in integrated learning at the interface between biomedical disciplines; and secondly, to support nonteaching focused, research experts, in the engaging delivery of lectures. Compared with traditional lecture delivery, students who had received Duet Lectorials reported an increase in their enjoyment of learning, a greater interest and engagement with the subject content, and, most importantly, improved in-depth understanding of the topic through an integrated perspective of the two disciplines. This positive outcome in student learning was further validated by improved performance in objective assessment tasks. Lecturers delivering Duet Lectorials reported a deepening of their own interdisciplinary knowledge that stimulated their enjoyment of teaching. Thus, interdisciplinary teaching using interactive Duet Lectorials emerges as a powerful approach to improve both student and teacher engagement and learning in the classroom, and brings a strong contribution to breaking the discipline-specific "silo" mentality in the teaching of complex aspects of biomedical sciences.
\end{abstract}

Keywords: Interdisciplinary Teaching; Duet Lectorial; Active Learning 


\section{Introduction}

In tertiary institutions, the biomedical disciplines have traditionally been taught from departments housing discipline-specific research activities. As research has progressed, the boundaries between disciplines have blurred, leading to an integrated approach to biomedical sciences, and ultimately making 'Personalized Medicine' a clear possibility (Salari, 2009). This development in integrated biomedical knowledge based on disciplines such as Genetics, Biochemistry, Pharmacology, Immunology, and Microbiology (to name a few) must now be included in tertiary education. Despite the need for interdisciplinary education, most tertiary institutions continue to educate students with discipline-specific knowledge delivered from within the rigid structures of departments (Salari, 2009).

Several significant challenges limit the ability of academics from discipline-specific departments to effectively teach in an interdisciplinary way. The first is cultural: the traditional resources available for teaching, including discipline-specific course 'ownership' driven by departmental funding structures and classic discipline-specific text-books as source material (Frodeman et al, 2017), do not facilitate inter-disciplinary teaching. Perhaps the most significant challenge, however, is the discipline-related language barrier that exists between disciplines after centuries of largely independent development, and the ensuing paucity of knowledge of academics in disciplines that are interconnected, but distinct, from that in which they have specialized during their research career.

To overcome the language barriers that have evolved within the silos of biomedical disciplines, and teach in an interdisciplinary way, we introduced Duet Lectorials.

\section{Methods}

\subsection{Format of Duet Lectorials}

Duet Lectorials utilized the discipline-specific knowledge and language proficiency of two experts straddling two related disciplines. The format was between that of a classic lecture and a tutorial, in which a three way discussion was initiated, firstly between each of the expert facilitators, and extended to the student cohort to participate in the sharing of ideas at the interface of the two disciplines. Three Duet Lectorials were assessed: The Immune System versus Malaria, The Immune System versus Herpesvirus, and The Immune System versus Cancer. In each case the discussion between the two discipline experts took the form of a 'battle', reflecting the real physiological battle that occurs at the interface between the two systems being studied. Students were thrown a 'catch box' carrying a microphone, through which they were encouraged to communicate their ideas on the 'weapons' each biological system may have evolved to be 'most fit' to survive the battle. 


\subsubsection{Assessment of the impact of Duet Lectorials on student experience}

Anonymous feedback was sought from 198 students who attended Malaria and Herpesvirus Duet Lectorials (136 and 126 respondents, respectively), and from 109 students who attended Cancer Duet Lectorials (70 respondents) over two academic years. Attendance at individual Duet Lectorials was awarded marks, but not compulsory. Students were classified into three groups according to whether they attended the duet lectorial (Attend), listened online to the recording but did not attend (Listen), or neither attended nor listened online, but read the lectorial notes provided only (Read). All students were asked to give a discrete response to statements as either Strongly Agree (SA), Agree (A), Neutral (N), Disagree (D), Strongly Disagree (SD). For simplicity, the analyses collated student responses into whether they agreed $(\mathrm{SA} / \mathrm{A})$ or did not agree $(\mathrm{N} / \mathrm{D} / \mathrm{SD})$ with the statements provided. Statistical analyses were performed using GraphPad Prism Fisher's exact test.

\subsubsection{Assessment of the impact of Duet Lectorials on student performance}

Performance was evaluated through objective assessment tasks based on Extended Matching Questions (EMQs) (Slattery, 2017). For the single lecturer cohort, the immunology expert taught all three lectures, and for the Duet Lectorials the same immunology expert was joined by experts in microbiology (Malaria and Herpesvirus Duet Lectorials) and cancer (Cancer Duet Lectorial). The EMQs were created by the immunologist; recording and notes were available for both traditional lectures and Duet Lectorials.

\section{Results}

\subsection{The Duet Lectorials were well structured}

Students were asked whether they preferred the structure of the Duet Lectorial, or that of standard lecture, for each Duet Lectorial they attended, listened to online, or read the notes only (Fig. 1). For each of the three Duet Lectorials, the vast majority of students preferred the Duet style of delivery. In the case of the Malaria Duet Lectorial, there was a significantly higher preference for the Duet structure of delivery by students who attended, compared with those who listened online only (Fig $1 \mathrm{~A}, * \mathrm{P}<0.05$ ). This was the first of the Duet Lectorials delivered, and we propose that actual attendance (rather than listening to a recorded session online) may have facilitated understanding because of additional visual and kinesthetic educational cues (Murphy et al, 2004). In the subsequent two Duet Lectorials, the lecturers were asked to slow the pace of the discussions to give students time to 'change gears' between the two discipline areas. There was no significant difference in the responses of students who attended versus those who listened online to the later Herpesvirus (Fig 1B) or Cancer (Fig1C) Duet Lectorials. In both of the latter cases, there 
was a significantly higher preference for the Duet structure of delivery by students who either attended or listened online to the material compared with the preference of students who read the notes only $(* * * \mathrm{P}<0.001)$. These data suggest that Duet Lectorials are most valuable in providing a setting in which students are actively participating in the learning environment.
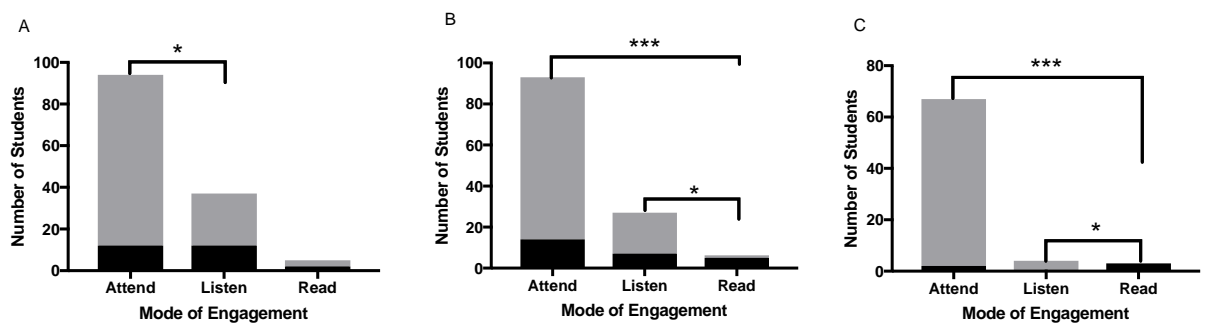

Figure 1. Students who attended or listened online preferred Duet Lectorials. Data representing (A) The Immune System versus Malaria Duet Lectorial $(n=136),(\boldsymbol{B})$ The Immune System versus Herpesvirus Duet Lectorial ( $n$ $126)$, and $(\boldsymbol{C})$ The Immune System versus Cancer Duet Lectorial (n=70). Students were asked if they preferred the structure of the Duet Lectorial (grey), or that of standard single lecturer delivery style (black), for each Duet Lectorial they attended. Statistical differences denoted as $* P<0.05$, ***P<0.001.

\subsection{The Duet Lectorial style was more enjoyable for students}

Students were asked whether they agreed or disagreed that Duet Lectorials were more fun than standard lectures (Fig. 2). For each of the three Duet Lectorials the vast majority of students agreed the Duet style of delivery was more enjoyable compared with the standard, single lecturer, delivery style. Here again the Malaria Duet Lectorial was enjoyed by students who attended the lectorial, compared with those who listened online only, consistent with the results in Section 3.1 (Fig $2 \mathrm{~A}, * \mathrm{P}<0.05$ ). Whereas, again, there was no significant difference in the number of students who enjoyed the Duet Lectorials between those who attended and listened online in the Herpes Duet Lectorial; the difference in enjoyment was seen only between those who did versus those who did not attend or listen to the lectorial (Fig $2 \mathrm{~B}, * * \mathrm{P}<0.01, * \mathrm{P}<0.05$ respectively). Since the majority of students attended the Cancer Duet Lectorial, the number of non-attending students was too small for meaningful comparison (Fig 2C).

\subsection{The Duet Lectorial style was more interesting and engaging for students}

Students were asked whether they agreed or disagreed that the Duet Lectorials were more interesting (Fig 3) and more engaging (Fig 4) than standard delivery, for each Duet Lectorial they attended, listened to online, or read the notes only. For each of the three Duet Lectorials the vast majority of students who attended or listened online agreed the Duet style of delivery was more interesting and engaging compared with the standard, single lecturer, delivery style. In contrast, students who read the notes only reported being 
significantly less interested (Fig $3 \mathrm{~A}, * * \mathrm{P}<0.01$; Fig $3 \mathrm{~B}, * * * \mathrm{P}<0.001$, $* * \mathrm{P}<0.01$ ) and engaged (Fig 4A, *P<0.05; Fig 4B, *** $<<0.001, * * \mathrm{P}<0.01$ ) with the Duet style of delivery.
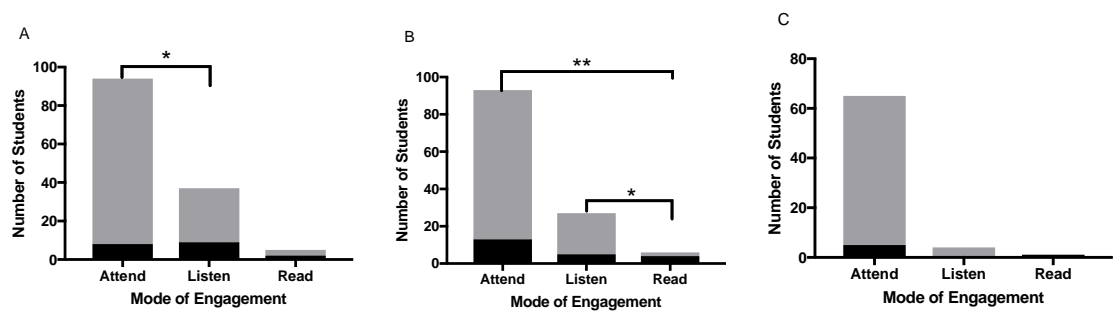

Figure 2. Duet Lectorials were more enjoyable for students who attended or listened online. Data representing (A) The Immune System versus Malaria Duet Lectorial $(n=136),(\boldsymbol{B})$ The Immune System versus Herpesvirus Duet Lectorial (n-126), and (C) The Immune System versus Cancer Duet Lectorial $(n=70)$. Students were asked whether they agreed (grey) or not (black) that Duet Lectorials were more fun than traditional lectures. Statistical differences denoted as $* P<0.05$, **P<0.01, ***P<0.001.
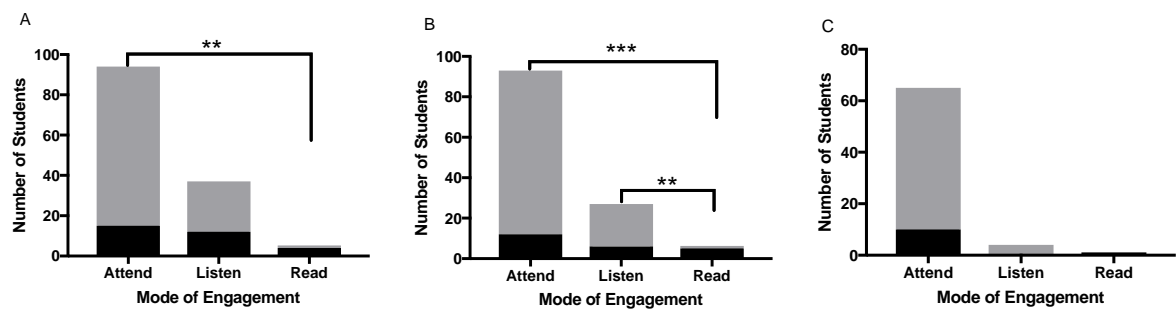

Figure 3. Duet Lectorials were more interesting for students who attended or listened. Data representing (A) The Immune System versus Malaria Duet Lectorial $(n=136),(\boldsymbol{B})$ The Immune System versus Herpesvirus Duet Lectorial (n-126), and (C) The Immune System versus Cancer Duet Lectorial (n=70). Students were asked whether they agreed (grey) or not (black) that Duet Lectorials were more interesting than traditional lectures. Statistical differences denoted as $* * P<0.01$, $* * * P<0.001$.
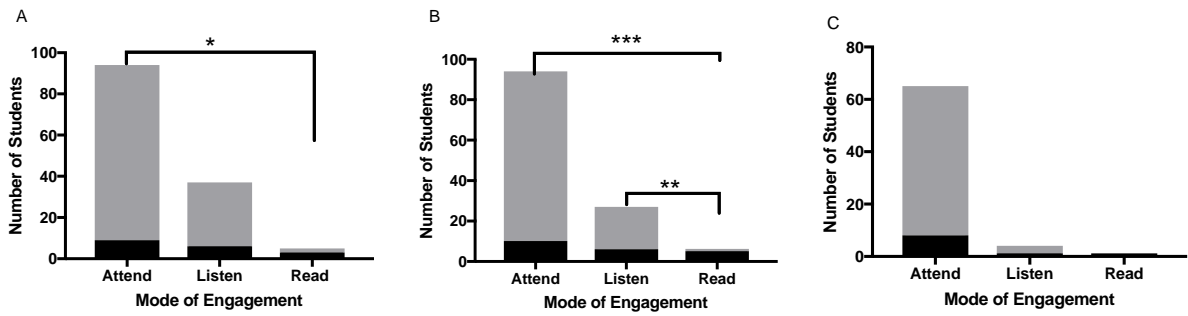

Figure 4. Duet Lectorials were more engaging for students who attended or listened. Data representing (A) The Immune System versus Malaria Duet Lectorial $(n=136),(\boldsymbol{B})$ The Immune System versus Herpesvirus Duet Lectorial (n-126), and (C) The Immune System versus Cancer Duet Lectorial ( $=70)$. Students were asked whether they agreed (grey) or not (black) that Duet Lectorials were more engaging than traditional lectures. Statistical differences denoted as $* P<0.05$, ***P<0.001. 


\subsection{The Duet Lectorial style assisted students to learn in an integrated way}

Students were asked whether they agreed (or not) that the Duet Lectorials assisted their integrated learning (Fig 5). For each of the three Duet Lectorials the vast majority of students who attended or listened online agreed the Duet style of delivery assisted their integrated learning compared with the standard, single lecturer, delivery style. However, again, the subset of students who only read the notes significantly less often reported improvement in their integrated learning (Fig 5A, $* \mathrm{P}<0.05$; Fig $5 \mathrm{~B}, * * \mathrm{P}<0.01, * \mathrm{P}<0.05$ ) with the Duet style.

The impact on learning was further assessed by comparing performance of students who were given interdisciplinary material, either using the standard lectures $(n=74)$, or using the Duet Lectorial ( $n=96$ ). The same objective assessment sheet (10 EMQs) (Slattery, 2017) was completed under examination conditions by both cohorts of students to assess their interdisciplinary knowledge of the interface between the immune system and malaria parasite biology. The students who received the Duet Lectorial significantly out-performed those who had received the standard lecture (mean mark of $65+/-22.2 \%$ versus $87+/$ $16.7 \%)$.
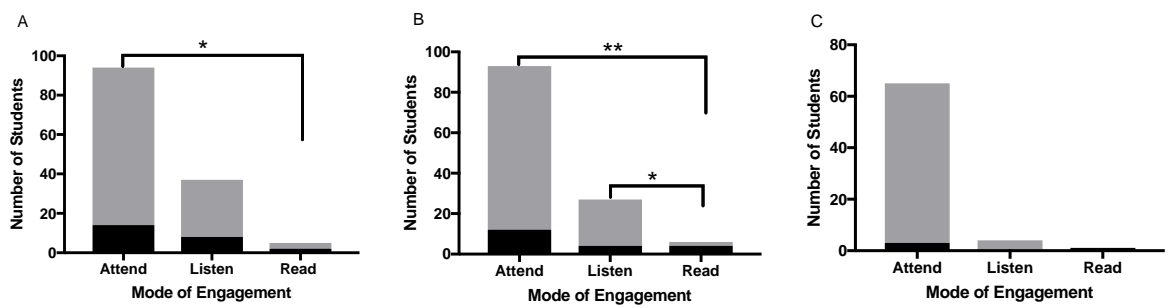

Figure 5. Duet Lectorials assisted students to learn in an integrated way. Data representing (A) The Immune System versus Malaria Duet Lectorial ( $n=136),(\boldsymbol{B})$ The Immune System versus Herpesvirus Duet Lectorial ( $n$ $126)$, and $(\boldsymbol{C})$ The Immune System versus Cancer Duet Lectorial $(n=70)$. Students were asked whether they agreed (grey) or not (black) that Duet Lectorials assisted their integrated learning more than traditional lectures. Statistical differences denoted as $* P<0.05$, **P<0.01. 

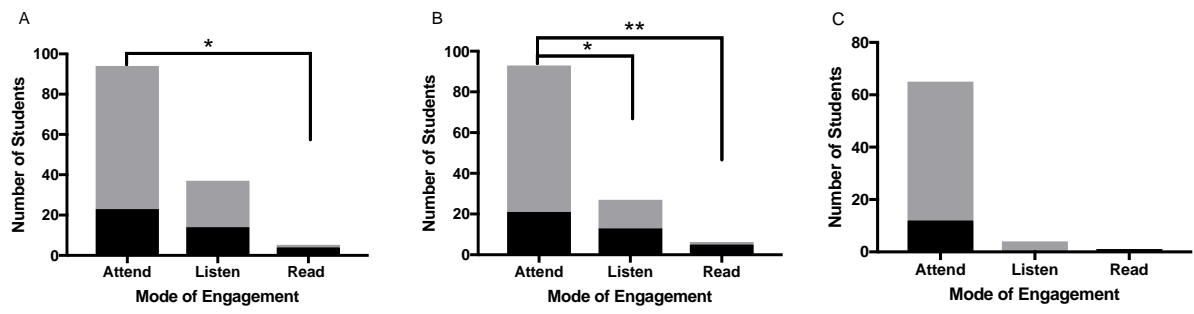

Figure 6. Students prefer to have more Duet Lectorials. Data representing (A) The Immune System versus Malaria Duet Lectorial (n=136), (B) The Immune System versus Herpesvirus Duet Lectorial (n-126), and (C) The Immune System versus Cancer Duet Lectorial $(n=70)$. Students were asked whether they would like to have more content delivered by the Duet Lectorial style (grey) or not (black) to replace traditional single lectures. Statistical differences denoted as $* P<0.05$, $* * P<0.01$.

\subsection{Students prefer to have more Duet Lectorial style delivery of material}

After each of three Duet Lectorials, students were asked whether they would like to have more of the course content delivered in the Duet Lectorial style, or have more standard lectures. Again the responses were stratified according to whether students attended, listened online, or read the notes only. The majority of students who attended or listened online preferred to have more Duet Lectorials, whereas, consistent with other parameters measured, significantly fewer students who neither attended nor listened online preferred to have more Duet Lectorial style delivery of material (Fig 6A, $* \mathrm{P}<0.05$; Fig $6 \mathrm{~B},{ }^{*} * \mathrm{P}<0.01$, $* \mathrm{P}<0.05)$.

\subsection{Benefits for the discipline-specific academics delivering the Duet Lectorials}

While the small number (3) of teachers involved in the study precludes meaningful statistical analyses of lecturer experience, anecdotal feedback was exclusively positive. Comments include: 'The Duet Lectorial offered me the opportunity to go in-depth in an area that is central to the infection process, but of which I had only largely superficial knowledge. This will certainly improve my own teaching on the subject'. 'The Duet Lectorial strengthened my ability to teach in an interactive way, not only with the other teacher, but also with the students, as the format is conducive to engaging the students to be 'on the side' of the pathogen. Altogether a very valuable and enjoyable experience.'

\section{Conclusions}

Duet Lectorials addressed the gap in interdisciplinary learning, and enhanced the communication style of non-teaching focused, research experts. This required a shift in pedagogical philosophy, from the traditional approach where a single lecturer is the discipline expert, to a synergistic approach, resembling research endeavors in which 
collaborative expertise is harnessed in an integrated way. It also required substantial extradisciplinary reading by both academics, to inform a script focussing on the interface between disciplines. The conversational style was experienced by both lecturers and students as a more natural mode of communication than is the case in a classical lecture (Mayer et al, 2004).

The impact of Duet Lectorials was overwhelmingly positive in all parameters measured. The vast majority of students who attended the event or listened to the content online preferred Duet Lectorials over traditional lectures, finding the approach to be more fun, interesting and engaging. These students reported an improvement in their integrated learning, which was substantiated by objective assessment through interdisciplinary EMQs under examination conditions. Finally, they expressed a strong preference for more Duet Lectorials to be included in their course; students who did not attend the Duet Lectorials were significantly less positive about the merits of the approach in all of the parameters tested. This suggest the benefits of this approach do not translate well to learning by reading notes, in isolation from active listening to the Duet Lectorial. It should be noted that the number of students who neither attended nor listened was small and may represent a self-selected sub-group that was disengaged from the subject material prior to its delivery (Massingham \& Herrington, 2006).

Teachers who delivered Duet Lectorials have requested further involvement in the program, which has enhanced their confidence in interdisciplinary teaching, and in turn had benefits in cross-fertilization of their research and in interdisciplinary grantsmanship. The successful uptake by students has prompted implementation of this approach in international postgraduate courses (Doerig \& Slattery, 2016, 2017), and for interdisciplinary research presentations at to the National Institute of Health (USA) (Cox \& Bainbridge, 2017).

The introduction of Duet Lectorials addressed a gap in interdisciplinary learning. The Duet Lectorials have enhanced the performance, enjoyment, interest and engagement of students and teachers alike. Overcoming the discipline language barriers and educating our students in an interdisciplinary way will not only prepare our graduates for the world of integrated medicine that awaits them, but also ultimately lead to changes in the culture of education within our universities, as these students become tomorrow's educators.

\section{References}

Salari K (2009) The Dawning Era of Personalized Medicine Exposes a Gap in Medical Education. PLoS Med 6(8): e1000138. 
Robert Frodeman, Julie Thompson Klein and Roberto Carlos Dos Santos Pacheco (2017) Administering Interdisciplinary Programs. The Oxford Handbook of Interdisciplinarity $2^{\text {nd }}$ Edition p538, Oxford University Press.

Slattery, R.M., (2017) Objective versus subjective methods to assess discipline-specific knowledge: a case for Extended Matching Questions (EMQs). 3rd International Conference on Higher Education Advances, HEAd'17

Murphy, R.J., Gray, S.A., Straja, S.R., and Bogert M.C. (2004) Student Learning Preferences and Teaching Implications Journal of Dental Education 68(8) 859-866

Mayer, R. E., Fennell, S., Farmer, L., \& Campbell, J. (2004). A Personalization Effect in Multimedia Learning: Students Learn Better When Words Are in Conversational Style Rather Than Formal Style. Journal of Educational Psychology, 96(2), 389-395.

Massingham, P. \& Herrington, T., (2006) Does Attendance Matter? An Examination of Student Attitudes, Participation, Performance and Attendance. Journal of University Teaching and Learning Practice 3(2)

Doerig, C.D., \& Slattery, R.M., (2016 \& 2017) The Immune System versus Malaria. Concepts in Parasitology Course, Kioloa, Australia. Australian Society for Parasitology.

Cox, B and Bainbridge, S (2017) The Human Placenta Project, National Institute of Child Health and Human Development (NICHD), National institute of Health (NIH) Natcher Conference Center. 